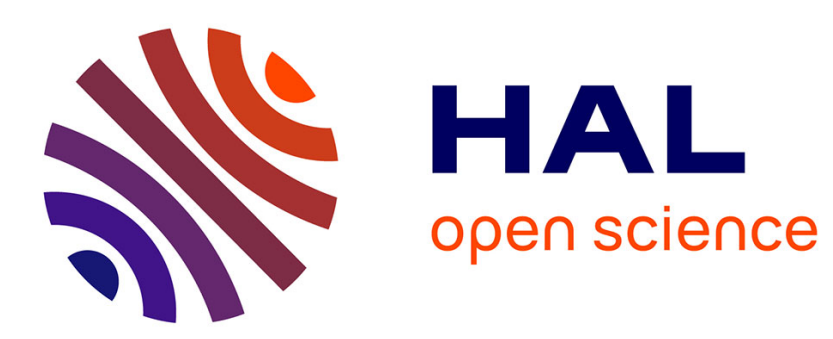

\title{
A finite element approximation for grazing collisions
}

\author{
Brigitte Lucquin-Desreux, Simona Mancini
}

\section{To cite this version:}

Brigitte Lucquin-Desreux, Simona Mancini. A finite element approximation for grazing collisions.

Transport Theory and Statistical Physics, 2003, 32, 3\& 4, pp.293-319. hal-00076838

\section{HAL Id: hal-00076838 \\ https://hal.science/hal-00076838}

Submitted on 24 Oct 2012

HAL is a multi-disciplinary open access archive for the deposit and dissemination of scientific research documents, whether they are published or not. The documents may come from teaching and research institutions in France or abroad, or from public or private research centers.
L'archive ouverte pluridisciplinaire $\mathbf{H A L}$, est destinée au dépôt et à la diffusion de documents scientifiques de niveau recherche, publiés ou non, émanant des établissements d'enseignement et de recherche français ou étrangers, des laboratoires publics ou privés. 
120024765_TT32_03-04_R1_071703

TRANSPORT THEORY AND STATISTICAL PHYSICS

Vol. 32, Nos. 3 \& 4, pp. 293-319, 2003

\title{
A Finite Element Approximation of Grazing Collisions
}

\author{
B. Lucquin-Desreux* and S. Mancini \\ Laboratoire Jacques-Louis Lions, \\ Université Pierre et Marie Curie, Paris, France
}

\begin{abstract}
In this article, we propose a finite element discretization of the Boltzmann-Lorentz operator for which it is possible to define a grazing collision limit. We illustrate this discretization by considering the evolution of a system of particles in a slab, subject to collisions both with the boundaries and with themselves. A comparison is made with isotropic collisions or with the Laplace-Beltrami operator. Moreover, in the case of multiplying boundary conditions, it is proven by numerical simulations the existence of a critical value for the absorption coefficient, which is independent on the grazing collision parameter. Finally, the focalization of a beam is studied and the numerical results are compared with previous simulations.
\end{abstract}

AMS Classification: $35 \mathrm{~B} 40 ; 65 \mathrm{~N} 30 ; 82 \mathrm{C} 40$.

*Correspondence: B. Lucquin-Desreux, Laboratoire Jacques-Louis Lions, Université Pierre et Marie Curie, Boite Courrier 187, 75252 Paris, Cedex 05, France; E-mail: lucquin@ann.jussieu.fr.

\section{3}

DOI: $10.1081 /$ TT-120024765

Copyright (c) 2003 by Marcel Dekker, Inc.

0041-1450 (Print); 1532-2424 (Online) www.dekker.com 


\section{INTRODUCTION}

Kinetic equations, as Boltzmann (or Fokker-Planck) equations, model the evolution of a system of particles subject to the action of a force field and to collisions. They are applied for example in semiconductor physics (see Markowich et al. (1994)), in the modeling of rarefied gases or plasmas (see Cercignani (1988)), in astrophysics (see Belleni-Morante and Moro (1996) for problems relative to interstellar clouds) or in biomathematics (see Arlotti et al. (2000) for the study of dynamics of populations). In the more general frame, the operator describing the scattering event is nonlinear and due to its complexity, many other models have been derived. Notably, the Lorentz operators, derived from the Boltzmann one or from the Fokker-Planck one, model the elastic collisions of particles against heaviest target ones (see Degond and Lucquin-Desreux (1996) and Lucquin-Desreux (2000) for the disparate masses particles asymptotics). In plasma physics for example, the Boltzmann-Lorentz operator represents (in first approximation with respect to the mass ratio) the effect of collisions due to neutral particles on the electron distribution function. On the other hand, the Fokker-Planck-Lorentz operator is the leading order term (still with respect to the mass ratio) describing the collisions of electrons against ions.

The Fokker-Planck operator is usually considered as an approximation of the Boltzmann collision operator when collisions become grazing, i.e., when the angle of deviation $\theta$ of a particle during a collision is very small. This essentially occurs for potentials of long range interaction, such as the Coulombian potential in the case of a plasma. This grazing collision limit of the Boltzmann operator towards the Fokker-Planck one has been proven in Degond and Lucquin-Desreux (1992) and Desvillettes (1992). In Desvillettes (1992), the scattering cross section is smooth; it depends on a small parameter $\varepsilon$ and it is localized around the value $\theta=0$ of the scattering angle when $\varepsilon$ goes to zero. On the other hand, the Coulomb case is studied in Degond (1992) by means of a quite different asymptotics: the scattering cross section has a nonintegrable singularity when the relative velocity of the colliding particles tends to zero but also when the scattering angle $\theta$ tends to zero. There is thus a necessary truncation of this angle around the value zero $(\theta \geq \varepsilon)$ and the small parameter $\varepsilon$ has a precise physical meaning: it is clearly identified as the plasma parameter. We also refer the reader to the works Goudon (1997a, 1997b) concerning the grazing collision limit of the Boltzmann operator when the scattering cross section corresponds to a inverse power force (i.e., $1 / r^{s}$, with $s>1$ ) and without angular cut-off. More recently, the convergence of Boltzmann-Lorentz operator towards the 
Fokker-Planck-Lorentz (or Laplace-Beltrami) operator in the so-called grazing collision limit has been proven in Buet et al. (2001), both for the asymptotics developed in Degond and Lucquin-Desreux (1992) and Desvillettes (1992).

In this article, we are concerned with the numerical approximation of the Boltzmann-Lorentz operator and with its grazing collision limit. Up to our knowledge, only few recent articless have been devoted to the study of the grazing collision limit at the discrete level: we can refer for example to Guérin and Méléard (2002) and Pareschi et al. (2002) concerning the full nonlinear Boltzmann operator discretized either by spectral methods (see Pareschi et al. (2002)) or by particle methods (see Guérin and Méléard (2002)). In our article, the considered scattering cross section is a simplified version of the one defined in Buet et al. (2001): it is assumed to be constant on a very small interval depending on the small parameter $\varepsilon$ and zero outside this interval. Our goal is to give a numerical approximation of the Boltzmann-Lorentz operator for grazing collisions for which it is possible to pass to the limit $\varepsilon \rightarrow 0$. We first decided to apply a finite element method (FEM) because this method is particularly well adapted to the limit problem (we refer for example to Cordier et al. (2000) for 3D computations). Secondly, in the FEM described below, we decided to do exact computations of the collision terms (instead of doing quadrature formulas): denoting by $h$ the velocity step, we can then choose $\varepsilon \leq h$ and thus study the limit $\varepsilon \rightarrow 0$ for a fixed discretization in velocity variable, i.e., for a fixed $h$ (which would not be possible when using quadrature formulas).

In order to show the efficiency of the proposed FEM approximation, we will consider a transport equation in which the collision operator is the Boltzmann-Lorentz operator for grazing collisions defined on a bounded region. Thus, we equip the kinetic equation with some kind of boundary conditions: in a first approach, we only take into account specular reflection boundary conditions. The numerical results obtained with the proposed FEM approximation are compared with those obtained considering the Laplace-Beltrami operator (i.e., its limit when $\varepsilon$ goes to zero), with the isotropic collisions (i.e., the scattering cross section is constant), and also with the collisionless case. Successively, we consider multiplying reflection boundary conditions (see for instance Belleni-Morante and Totaro (1996) and Mancini and Totaro (1998)): each particle colliding on the boundary of the considered region is reflected and multiplied by a factor bigger than one. In this optic, as the region where the evolution of particles takes place is bounded, it seems physically necessary to consider also an absorption term in our transport equation. The existence and uniqueness of the solution of such a problem, with the absorption cross section $\sigma$ bigger 
than a theoretical value $\sigma_{t}$, has been proven in Belleni-Morante (1996) for multiplying Maxwell boundary conditions, and in Mancini and Totaro (1998) in the general case. We will numerically prove the existence of a critical absorption cross section $\sigma_{n}<\sigma_{t}$ for which the total number of particles does not sharply increase nor vanishes with time. Moreover, we also remark that both $\sigma_{n}$ and the equilibrium profile of the particle density do not depend on the initial data.

The article is organized as follows. In Sec. 2, we present the finite element approximation for the Boltzmann-Lorentz operator for grazing collisions and also for isotropic collisions. In Sec. 3, we present the test problems used to validate our numerical approximation. Section 4 concerns the results of the numerical simulations. Finally, in Sec. 5, we give some concluding remarks.

\section{NUMERICAL SCHEMES}

The goal of this article is the numerical approximation of the Boltzmann-Lorentz operator for grazing collisions. We recall that the Boltzmann-Lorentz operator models for example the elastic collisions of a heavy particle against a light one (see Degond and Lucquin-Desreux, (1996) and Lucquin-Desreux (2000)). Thus, it acts only on the velocity direction $\theta$ of the particles, leaving unchanged the velocity modulus $|v|$ (which we will consider normalized to one). In the bi-dimensional case, the Boltzmann-Lorentz operator is given by:

$$
Q(f)(\theta)=\int_{S^{1}} B\left(\theta-\theta^{\prime}\right)\left(f\left(\theta^{\prime}\right)-f(\theta)\right) d \theta^{\prime},
$$

where $B\left(\theta-\theta^{\prime}\right)$ is the scattering cross section which represents the probability for a particle entering a collision with velocity direction $\theta^{\prime}$ to out-go the scattering event with a velocity direction $\theta$. For instance, $B\left(\theta-\theta^{\prime}\right)$ may be determined from the interaction force between the particles (see Degond and Lucquin-Desreux (1992), Desvillettes (1992), and Goudon (1997b)); in particular, we recall that, in the case of isotropic collisions, it does not depend on $\theta$ nor on $\theta^{\prime}$.

We are interested in grazing collisions, i.e., we want to take into account also the very small deviations in the trajectories of the particles. This fact is emphasized by introducing the dependence of the scattering cross section $B$ on a small parameter $\varepsilon$. Recently, Buet, Cordier, and Lucquin-Desreux (see Buet et al. (2001)) have proven the convergence, when the small parameter $\varepsilon$ tends to zero, of the Boltzmann-Lorentz 
operator for grazing collisions towards the Fokker-Planck-Lorentz operator, which is simply the Laplace-Beltrami operator.

Our goal here is to give a numerical approximation of the Boltzmann-Lorentz operator for which it is possible to pass to the limit $\varepsilon \rightarrow 0$ for a fixed velocity discretization. As shown in the sequel, this is possible when applying for example a finite element approach, where the integral terms are all exactly computed. The numerical results we obtain are then compared with the collisionless case, the case of isotropic collisions, and those obtained when considering the Laplace-Beltrami operator.

\subsection{An Implicit Finite Element Approximation}

We want to approximate by means of a finite element method the following Cauchy problem:

$$
\left\{\begin{array}{l}
\frac{\partial f}{\partial t}=Q(f), \\
f(t=0)=f^{0},
\end{array}\right.
$$

where $Q(f)$ is defined by Eq. (1). We note that the dependence of the density $f$ on the space variable is not underlined in this paragraph because the position of the particles does not play any role during a collision. The angle variable $\theta$ belongs to the unit circle $S^{1}$, i.e., $\theta \in[-\pi, \pi]$ and the distribution function $f$ satisfies periodic boundary conditions.

We first consider the following piecewise affine approximation $f^{N_{\theta}}$ of the function $f$ :

$$
f(t, \theta) \simeq f^{N_{\theta}}(t, \theta)=\sum_{j=0}^{N_{\theta}-1} \varphi_{j}(\theta) f_{j}(t)
$$

where $\varphi_{j}, j \in\left\{0, \ldots, N_{\theta}-1\right\}$, are the classical "hat functions" of the $P^{1}$ finite element approximation. More precisely, denoting by $\theta_{j}=-\pi+h j$, with $h=2 \pi / N_{\theta}$, we have $\varphi_{i}\left(\theta_{j}\right)=\delta_{i j}$ (=1 if $\mathrm{i}=\mathrm{j}$ and 0 otherwise), so that $f_{j}(t)=f^{N_{\theta}}\left(t, \theta_{j}\right)$.

The weak discretized form of Eq. (2) then reads:

$$
\begin{aligned}
& \sum_{j=0}^{N_{\theta}-1} \frac{\partial f_{j}}{\partial t} \int_{S^{1}} \varphi_{j}(\theta) \varphi_{i}(\theta) d \theta \\
& \quad=\sum_{j=0}^{N_{\theta}-1} f_{j} \int_{S^{1}} \int_{S^{1}} B\left(\theta-\theta^{\prime}\right)\left[\varphi_{j}\left(\theta^{\prime}\right) \varphi_{i}(\theta)-\varphi_{j}(\theta) \varphi_{i}(\theta)\right] d \theta^{\prime} d \theta .
\end{aligned}
$$


Setting:

$$
\left\{\begin{array}{l}
A_{i j}=\int_{S^{1}} \varphi_{i}(\theta) \varphi_{j}(\theta) d \theta, \\
Q_{i j}=\int_{S^{1}} \int_{S^{1}} B\left(\theta-\theta^{\prime}\right)\left[\varphi_{i}(\theta) \varphi_{j}\left(\theta^{\prime}\right)-\varphi_{i}(\theta) \varphi_{j}(\theta)\right] d \theta d \theta^{\prime},
\end{array}\right.
$$

we write the discretized problem more compactly as follows:

$$
\sum_{j=0}^{N_{\theta}-1} \frac{\partial f_{j}}{\partial t} A_{i j}=\sum_{j=0}^{N_{\theta}-1} f_{j} Q_{i j} .
$$

The matrix $A$ with entries $A_{i j}$ is the so called "mass matrix," while the matrix $Q$ with entries $Q_{i j}$ represents the collision matrix. In the next paragraph, we give the expression of these two matrices in the context of grazing collisions; the detailed computations are performed in the Appendix A1. Before this, let us precise the time discretization of Eq. (4). In order to avoid a limitation of the time step $\Delta t$ in terms of the angular step $\Delta \theta=h$, we will use a fully implicit scheme. We introduce some notations. Let us denote by $F^{n}$ the $N_{\theta}$ dimensional vector with entries $F_{j}^{n}=f\left(n \Delta t, \theta_{j}\right)$, for $j \in\left\{0, \ldots, N_{\theta}-1\right\}$. The implicit finite element scheme we consider reads:

$$
\left\{\begin{array}{l}
F^{n+1}=(A-\Delta t Q)^{-1} A F^{n}, \quad n \geq 0 \\
F^{0}=\left(F_{i}^{0}\right)_{0 \leq j \leq N_{\theta}-1},
\end{array}\right.
$$

with $F_{j}^{0}=f^{0}\left(\theta_{j}\right)$.

\subsection{Grazing Collisions}

In the case of grazing collisions, the scattering cross section $B$ is localized and depends on a small parameter $\varepsilon$. In the nonCoulombian case, $B$ has the following expression (see Buet et al. (2001) and Desvillettes (1992)):

$$
B^{\varepsilon}(z)=\frac{1}{\varepsilon^{3}} \bar{B}(z / \varepsilon) \chi_{S^{1}}(z / \varepsilon)
$$

where $\chi$ denotes the characteristic function (i.e., $\chi_{S^{1}}(z)=1$ if $z \in S^{1}$ and 0 otherwise). We will here consider a simplified model for which the scattering cross section is given by:

$$
B^{\varepsilon}(z)= \begin{cases}B_{0} & \text { if }|z|<\varepsilon \pi \\ 0 & \text { else }\end{cases}
$$


with $B_{0}=1 / \varepsilon^{3}$. We then have:

Proposition 2.1. Setting $\alpha=\varepsilon \pi / h$ and assuming that $\alpha \leq 1$, the grazing collision matrix $Q$ is given by:

$$
Q=\frac{\pi^{3}}{3 h}\left(\begin{array}{cccccccc}
-2+\frac{3}{4} \alpha & 1-\frac{1}{2} \alpha & \frac{1}{8} \alpha & 0 & \ldots & 0 & \frac{1}{8} \alpha & 1-\frac{1}{2} \alpha \\
1-\frac{1}{2} \alpha & \ddots & \ddots & \ddots & \ddots & \ddots & \ddots & \frac{1}{8} \alpha \\
\frac{1}{8} \alpha & \ddots & \ddots & \ddots & \ddots & \ddots & \ddots & 0 \\
0 & \ddots & \ddots & \ddots & \ddots & \ddots & \ddots & \vdots \\
\vdots & \ddots & \ddots & \ddots & \ddots & \ddots & \ddots & 0 \\
0 & \ddots & \ddots & \ddots & \ddots & \ddots & \ddots & \frac{1}{8} \alpha \\
\frac{1}{8} \alpha & \ddots & \ddots & \ddots & \ddots & \ddots & \ddots & 1-\frac{1}{2} \alpha \\
1-\frac{1}{2} \alpha & \frac{1}{8} \alpha & 0 & \ldots & 0 & \frac{1}{8} \alpha & 1-\frac{1}{2} \alpha & -2+\frac{3}{4} \alpha
\end{array}\right) .
$$

Proof. The proof is detailed in the Appendix A1.

By means of the same arguments applied in order to compute the matrix $Q$ (see Appendix A1), we have that the mass matrix $A$ reads:

$$
A=\frac{h}{3}\left(\begin{array}{cccccc}
2 & \frac{1}{2} & 0 & \ldots & 0 & \frac{1}{2} \\
\frac{1}{2} & \ddots & \ddots & \ddots & \ddots & 0 \\
0 & \ddots & \ddots & \ddots & \ddots & \vdots \\
\vdots & \ddots & \ddots & \ddots & \ddots & 0 \\
0 & \ddots & \ddots & \ddots & \ddots & \frac{1}{2} \\
\frac{1}{2} & 0 & \ldots & 0 & \frac{1}{2} & 2
\end{array}\right) .
$$

We remark that if we let $\varepsilon \rightarrow 0$ then $Q_{i j} \rightarrow\left(h \pi^{3}\right) /\left(3 D_{i j}\right)$, where $D_{i j}$ is the classical finite difference discretization of the Laplace-Beltrami operator $\Delta f=\partial_{\theta \theta} f$ :

$$
D=\frac{1}{h^{2}}\left(\begin{array}{cccccc}
-2 & 1 & 0 & \ldots & 0 & 1 \\
1 & \ddots & \ddots & \ddots & \ddots & 0 \\
0 & \ddots & \ddots & \ddots & \ddots & \vdots \\
\vdots & \ddots & \ddots & \ddots & \ddots & 0 \\
0 & \ddots & \ddots & \ddots & \ddots & 1 \\
1 & 0 & \ldots & 0 & 1 & -2
\end{array}\right) .
$$




\subsection{Isotropic Collisions}

In the following simulations, in order to compare the evolution of the system of particles with grazing collisions and with isotropic collisions, we will not compute the exact solution for the isotropic Boltzmann-Lorentz operator (although it is possible), but we will approximate the isotropic operator following the same discretization as before.

Recalling that for isotropic collisions the scattering cross section $B$ is a constant (which we will assume equal to one), we get the following finite element discretization of the isotropic Lorentz operator. The gain matrix $G$ is given by: $G=\left(G_{i j}\right)_{0 \leq i, j \leq N_{\theta}-1}$, with $G_{i j}=h^{2}$, whereas, the loss matrix $L$ is such that: $L=2 \pi A$. Then we can apply the implicit finite element scheme (5), where now the matrix $Q=G-L$ represents the $P^{1}$ finite element approximation of the isotropic Lorentz operator.

\section{THE TEST PROBLEM}

We illustrate the finite element approximation for the Boltzmann-Lorentz operator with grazing collisions on the following transport equation:

$$
\partial_{t} f+\cos \theta \partial_{x} f+\sigma f=\frac{1}{\tau} Q(f)
$$

This transport type equation describes the evolution of a system of particles moving between two parallel plates (slab), subject to absorption by the host medium and to elastic collisions with other particles present in the slab. We recall that $f=f(x, \theta, t)$ represents the number of particles which at time $t \geq 0$, are in the position $x \in[-L,+L]$ with velocity $v=(\cos \theta, \sin \theta), \theta \in[-\pi, \pi]$. Moreover, $Q(f)$ is the Lorentz collision operator defined by Eq. (1), $\sigma \geq 0$ is the absorption cross section, and $\tau$ is the relaxation time; this one is assumed to be equal to one, except in the last numerical test concerning the defocusing of a beam (see Sec. 4.4 below and Cordier et al. (2000)).

The interactions of the particles with the two plates of the slab may be described by means of a linear, bounded, and positive operator 
$\Lambda: \Gamma^{\text {out }} \rightarrow \Gamma^{\text {in }}$ which relates the incoming and outgoing flux of particles as follows:

$$
f^{\text {in }}=\Lambda f^{\text {out }} .
$$

In Eq. (9), $f^{\text {in }}$ and $f^{\text {out }}$ are the traces of the distribution function respectively to the incoming and outgoing boundary sets:

$$
\begin{aligned}
\Gamma^{\text {in }} & =\{(-L, \theta), \cos \theta>0\} \times\{(L, \theta), \cos \theta<0\}, \\
\Gamma^{\text {out }} & =\{(L, \theta), \cos \theta>0\} \times\{(-L, \theta), \cos \theta<0\} .
\end{aligned}
$$

We define the norms on the incoming and outgoing sets as usual:

$$
\|f\|_{\text {out }}=\int_{(x, \theta) \in \Gamma_{\text {out }}} f(x, \theta)|\cos \theta| d \theta, \quad\|f\|_{\text {in }}=\int_{(x, \theta) \in \Gamma^{\text {in }}} f(x, \theta)|\cos \theta| d \theta,
$$

and we consider the norm for the operator $\Lambda$ to be given by:

$$
\|\Lambda\|=\max _{\left\|f^{\text {out }}\right\|_{\text {out }}=1}\left\|\Lambda f^{\text {out }}\right\|_{\text {in }} .
$$

We will consider multiplying boundary conditions (see Mancini and Totaro (1998)), i.e., the operator $\Lambda$ is such that $\left\|\Lambda f^{\text {out }}\right\|_{\text {in }} \geq c\left\|f^{\text {out }}\right\|_{\text {out }}$, with $c>1$. In other words, a multiplication of particles takes place on the boundaries. For instance, the boundary operator $\Lambda$ may represent reflection, or diffusion boundary conditions, as well as a linear combination of them. In the following simulations, we will consider multiplying reflective boundary conditions:

$$
\begin{cases}f(-L, \theta)=\alpha_{L} f\left(-L, \theta^{\prime}\right), & \text { for } \cos \theta>0, \\ f(L, \theta)=\alpha_{R} f\left(L, \theta^{\prime}\right), & \text { for } \cos \theta<0,\end{cases}
$$

with $\theta^{\prime}=\pi-\theta$, where $\alpha_{L}$ and $\alpha_{R}$ respectively denote the reflection coefficients on the left and right boundaries of the slab (i.e., on $x=-L$ and $x=L$ respectively). As the region where the evolution takes place is bounded, it is natural to consider also an absorption term $\sigma f$ in order to avoid the growth of the number of particles (see the results of Fig. 7 below). Summarizing, the problem which we approximate numerically reads as follows:

$$
\left\{\begin{array}{l}
\partial_{t} f+\cos \theta \partial_{x} f+\sigma f=\tau^{-1} Q(f), \\
f^{\text {in }}=\Lambda f^{\text {out }} \\
f(x, \theta, 0)=f_{0}(x, \theta) .
\end{array}\right.
$$

It is clear that the efficiency of the absorption coefficient $\sigma$ will depend on the "power" of the multiplication coefficient (i.e., the norm of the operator $\Lambda$ ), on the modulus of the particle velocities $|v|$ (here 
equal to 1) and on the dimension of the considered region (here the distance $2 L$ between the two plates). It has been proven in Mancini and Totaro (1998), by means of the semi-groups theory and for a fixed $\varepsilon$, that if $\|\Lambda\| \leq 1$, then problem (11) admits a unique solution whatever is $\sigma \geq 0$; whereas, if $\|\Lambda\|>1$, then problem (11) admits a unique solution provided that:

$$
\sigma>\frac{|v| \ln \|\Lambda\|}{2 L}=\sigma_{t}
$$

$\sigma_{t}$ is called the theoretical critical absorption cross section. In the particular case of multiplying reflective boundary conditions (10), the theoretical value for the critical absorption cross section is given by (see Belleni-Morante and Totaro (1996)):

$$
\sigma_{t}=\frac{|v| \ln \left(\max \left\{\alpha_{L}, \alpha_{R}\right\}\right)}{2 L} .
$$

We will numerically compute a critical value $\sigma_{n}$ for the absorption cross section and we will precise the behavior of the solution of problem (11) in terms of the absorption coefficient $\sigma$ : we will see that, if $\sigma<\sigma_{n}$ the particle density significantly grows in a finite time, for $\sigma>\sigma_{n}$ the particle density rapidly goes to zero in a finite time, and for $\sigma=\sigma_{n}$ it remains bounded without vanishing (see Sec. 4.3 below). This numerical critical value $\sigma_{n}$ does not depend on $\varepsilon$; moreover, it is smaller than the theoretical one $\sigma_{t}$. This fact does not contradict at all the results proven in Belleni-Morante and Totaro (1996) or Mancini and Totaro (1998); in fact, it just shows the existence of a solution also for absorption cross sections $\sigma \in] \sigma_{n}, \sigma_{t}\left[\right.$ (since $\sigma_{n}<\sigma_{t}$ ), which was not possible to prove by means of the semigroup theory.

\section{NUMERICAL SIMULATIONS}

In order to simulate the evolution problem (11), we apply a first order splitting in time method. First, we approximate by means of an upwind explicit finite difference method the following transport problem (see paragraph 4.1 below):

$$
\left\{\begin{array}{l}
\frac{\partial f}{\partial t}+\cos \theta \frac{\partial f}{\partial x}+\sigma f=0 \\
f^{\text {in }}=\Lambda f^{\text {out }} \\
f(x, \theta, 0)=f_{0}(x, \theta) .
\end{array}\right.
$$


Second, we apply the implicit finite element scheme of Sec. 2.1 to the pure collision problem:

$$
\left\{\begin{array}{l}
\frac{\partial f}{\partial t}=\tau^{-1} Q f \\
f(x, \theta, 0)=f_{0}(x, \theta) .
\end{array}\right.
$$

At each iteration, the program solves separately the transport and collision parts. Our whole numerical scheme is first order in all variables. We have done this choice in order to simplify the presentation of the method, but it would be naturally possible to improve the accuracy of the method by using appropriate second order schemes for each step (convection, collision) coupled with a second order (of Strang type) splitting algorithm.

We will here perform essentially three different numerical tests. In the first two ones, developed in paragraphs 4.2 and 4.3 below, the relaxation time $\tau$ is equal to one. In paragraph 4.2 , we essentially focalize on the discrete collision operator itself: the boundary conditions are classical specular reflection, and there is no absorption; the grazing collision operator is then compared to the Laplace-Beltrami operator (i.e., to its limit when $\varepsilon$ goes to zero), to the isotropic operator and also to the collisionless case. The dependence of the solution on the grazing parameter $\varepsilon$ is also numerically investigated, first for $\varepsilon \leq h / \pi$, but also for bigger values of $\varepsilon$. The influence of multiplicative boundary conditions is then studied in paragraph 4.3: in particular, the existence of a numerical critical absorption cross section, which does not depend on $\varepsilon$ (for sufficiently small values of $\varepsilon$ ) nor on the initial data is shown. Moreover, we underline the existence of a unique profile of equilibrium (independent on $\varepsilon$ and on the initial data) for the particle density. Finally, in paragraph 4.4, we study the influence of the time relaxation $\tau$ for a photonic type problem already considered in a previous work (see Cordier et al. (2000)). This last test is further investigated in Cordier et al. (2002). Let us firstly precise the numerical scheme used to approximate the convective part of the equation, i.e., problem (14).

\subsection{Transport Problem Approximation}

We consider $N_{x}(=50$ in simulations, unless otherwise specified $)$ discretization points $x_{i}, \quad i \in\left\{1, \ldots, N_{x}\right\}$, for the space variable $x \in[-L, L]$ (with $L=1)$ and $N_{\theta}(=50$ in simulations, unless otherwise specified) discretization points for the angle variable $\theta \in S^{1}$. We introduce the following symmetric discretization of the circle $S^{1}: \theta_{j}=-\pi+h j$ with 
$h=2 \pi / N_{\theta}$ and $j=0, \ldots, N_{\theta}-1$. We have that if $\cos \theta_{j}$ is a discretization point, then also $-\cos \theta_{j}$ is one. Moreover, if $j$ and $j^{\prime}$ are such that $j^{\prime}=s(j)$, where $s(j)=\left(N_{\theta} / 2-j\right) \bmod (N)$ (see Fig. 1), then: $\cos \theta_{j^{\prime}}=-\cos \theta_{j}$.

Considering the above discretization, we approximate the transport problem (14) by means of an upwind explicit finite difference scheme as follows:

- If $\cos \theta_{j}>0$ then,

$$
f_{1, j}^{n+1}=\alpha_{L} f_{1, s(j)}^{n+1}
$$

and for $i=2 \ldots N_{x}$

$$
f_{i j}^{n+1}=\left(1-(\Delta t / \Delta x) \cos \theta_{j}-\sigma \Delta t\right) f_{i j}^{n}+(\Delta t / \Delta x) \cos \theta_{j} f_{i-1, j}^{n} .
$$

- If $\cos \theta_{j}<0$ then

$$
\begin{aligned}
f_{N_{x}, j}^{n+1} & =\alpha_{R} f_{N_{x}, s(j)}^{n+1} \\
\text { and for } i & =1 \ldots N_{x}-1 \\
f_{i j}^{n+1} & =\left(1+(\Delta t / \Delta x) \cos \theta_{j}-\sigma \Delta t\right) f_{i j}^{n}-(\Delta t / \Delta x) \cos \theta_{j} f_{i+1, j}^{n} .
\end{aligned}
$$

We recall that $f_{i j}^{n}=f\left(x_{i}, \cos \theta_{j}, t_{n}\right)$ is the value of the distribution function $f$ at the position $x_{i}=-L+\Delta x(i-1), i=1 \ldots N_{x}$, and $\Delta x=2 L /\left(N_{x}-1\right)$, with velocity $\cos \theta_{j}, j=0 \ldots N_{\theta}-1$, and at time $t_{n}=n \Delta t, n=1 \ldots N_{\text {iter }}$.

Setting $\lambda=\Delta t / \Delta x$, the CFL condition writes (for any $\sigma \geq 0$ ): $\lambda \leq 1$. In the simulations below, $\lambda$ is taken equal to 0.8 (but $\lambda=1$ would give exactly the same results). Moreover, we choose $\alpha_{L}=2 \alpha_{R}=4$ and $\varepsilon=0.001$, unless otherwise specified.

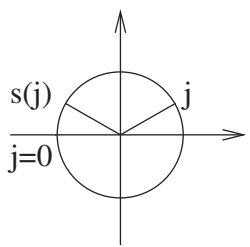

Figure 1. Discretization on the sphere.

\section{F1}




\subsection{Grazing Collisions and Comparison with Other Collision Terms}

In this section we present numerical results concerning the grazing collision limit at the discrete level and also a comparison with different types of collisions. We take into account only specular reflection boundary conditions (i.e., $\alpha_{L}=\alpha_{R}=1$ ), the absorption cross section is given by $\sigma=0$ (i.e., the number of particles is constant along all the evolution), and the relaxation time is $\tau=1$. We introduce the density function:

$$
n(x, t)=\int f(x, \theta, t) d \theta
$$

and the number of particles inside the slab at time $t$ :

$$
N(t)=\int_{-L}^{+L} n(x, t) d x .
$$

In Fig. 2, we show how the behavior of the density $n(x, t)$ changes when changing the value of the grazing parameter $\varepsilon$. We have first considered values of $\varepsilon$ such that $\varepsilon \leq h / \pi$, but also larger values of $\varepsilon$ : in this last case,

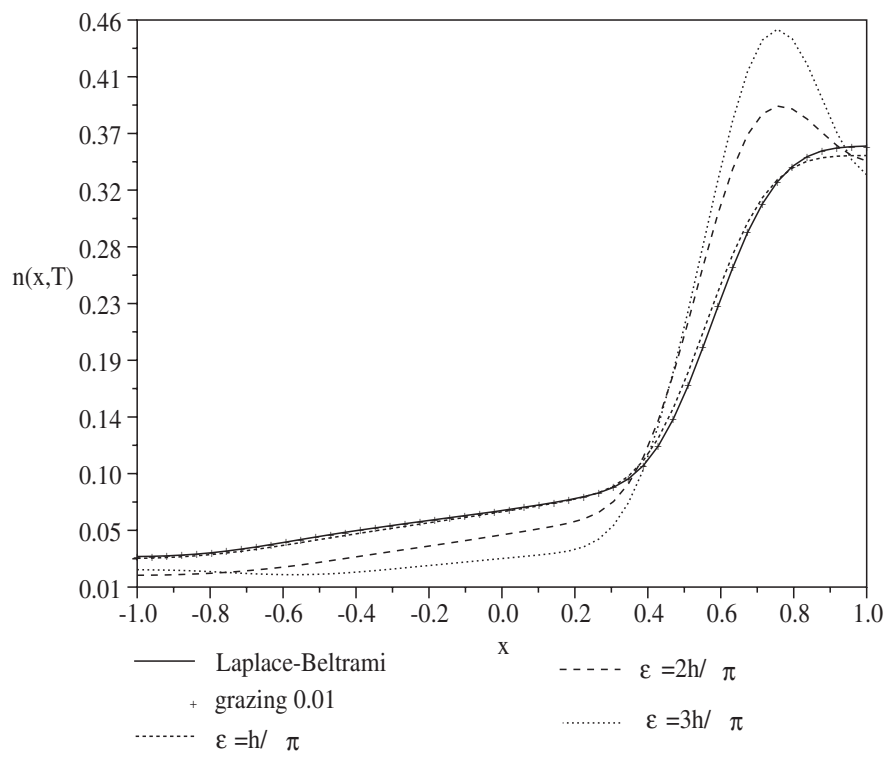

Figure 2. The density $n(x, T)$, with $\sigma=0, \alpha_{R}=\alpha_{L}=1$, for different values of $\varepsilon$ : $\varepsilon=10^{-2}, h / \pi, 2 h / \pi, 3 h / \pi$ and for the Laplace-Beltrami operator, with initial data given by a Dirac, $t=\Delta t N_{\text {iter }}$, with $N_{\text {iter }}=100, N_{x}=50, N_{\theta}=10$. 
547 the collision terms have been computed by use of standard first order quadrature formulas. In particular, we note that for $\varepsilon<10^{-2}$ the difference between the grazing collision case and the Laplace-Beltrami operator is too small to be observed. In fact, the difference is of order $\varepsilon h$, so that it is rapidly very small for small values of $\varepsilon$ (and for small $h$ as well). In order to precise this behavior, we have computed in Fig. 3 the $L^{\infty}$ norm of the relative error between the density $n_{\varepsilon}$ of the grazing collision problem and that of the limiting one, denoted by $n_{L B}$, i.e.,

$$
\max _{i}\left(\frac{n_{\varepsilon}(i, T)-n_{L B}(i, T)}{n_{L B}(i, T)}\right),
$$

in terms of $\varepsilon$ and for different values of $h$ (with $T=50 \Delta t$ ). We first remark that this error is very small for $\varepsilon=10^{-3}$. But as expected, we observe a difference between the case $\varepsilon=h / \pi$ and $\varepsilon=10^{-2}$ for large values of $h(h=\pi / 2)$, while this difference decreases for smaller values of $h$. Figure 2 is related to the "medium" case $h=\pi / 5$ (i.e., $N_{\theta}=10$ ).

In Fig. 4, we compare four types of collisions: the collisionless case $(Q=0)$, the case of isotropic collisions (with $B=1 / 4 \pi$ in order to fit the first nonzero eigenvalues), the grazing collisions case (with $\varepsilon=10^{-3}$ ) and its limit when $\varepsilon \rightarrow 0$, i.e., the Laplace-Beltrami operator. We trace the evolution of the density $n(\cdot, T)$ for four different times of computation $T=\Delta t N_{\text {iter }}$, with $N_{\text {iter }}=50,100,150,200$. We observe that the grazing collisions case and the Laplace-Beltrami operator give the same results (up to a difference of order $10^{-4}$ which is not noticeable on the figure).

Naturally, in all cases, we have checked numerically that the total density $N(t)$ defined by Eq. (18) was constant with respect to time.

\subsection{Absorption Cross Section and Multiplying Boundary Conditions}

Still assuming $\tau=1$, we now take into account multiplying boundary conditions, in particular $\alpha_{L}=2, \alpha_{R}=4$. We consider two initial

\begin{tabular}{|l|l|l|l|}
\hline & $N_{\theta}=4$ & $N_{\theta}=10$ & $N_{\theta}=20$ \\
\hline$\varepsilon=h / \pi$ & 0.15639 & 0.03291 & 0.01392 \\
\hline$\varepsilon=10^{-2}$ & 0.00269 & 0.00158 & 0.00136 \\
\hline$\varepsilon=10^{-3}$ & 0.00027 & 0.00016 & 0.00013 \\
\hline
\end{tabular}

Figure 3. Influence of parameters $\varepsilon$ and h. 

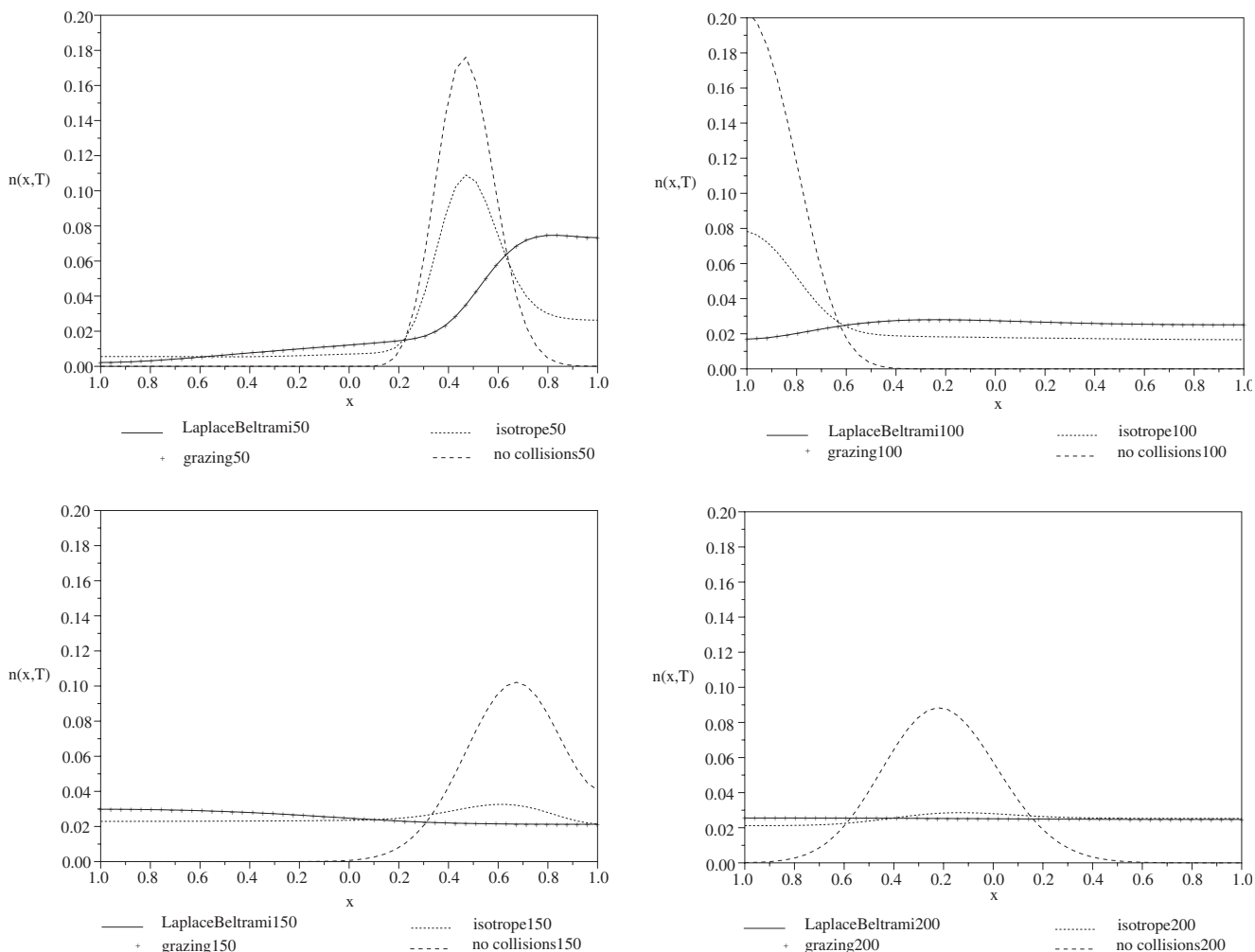

Figure 4. The density $n(\cdot, T)$, with $\sigma=0, \alpha_{R}=\alpha_{L}=1$, for the grazing collisions with $\varepsilon=0.001$,

the Laplace-Beltrami operator, the isotropic collisions case, and the collisionless case, with initial data given by a Dirac, $T=\Delta t N_{\text {iter }}$, with $N_{\text {ittr }}=50$ (top on the left), 100 (top on the right), 150 (bottom on the left), 200 (bottom on the right). 
data: first, a constant function both in space and velocity; second, a Dirac mass both in space and velocity.

In Fig. 5, we show the convergence of the sequence of critical absorption cross sections towards a finite value $\sigma_{n}$ for $\varepsilon=0.001$. The critical absorption value we compute is independent (for large times) on the initial data: in fact we observe that both the constant initial data and the Dirac mass give the same value. Moreover, this value is smaller than the theoretical one $\sigma_{t}$ given by Eq. (13). The way the computation is performed is the following one:

- We compute the total densities $N\left(t_{i}, \sigma\right)$ for $t_{i}=(i+1) 50 \Delta t$, with $i=0, \ldots, 9$, and for different values of $\sigma$.

- We determine the intersection point of two successive curves $N\left(t_{i}, \cdot\right)$ and $N\left(t_{i+1}, \cdot\right)$ describing the evolution of the density in terms of $\sigma$; the respective value of $\sigma$ for the intersection points are called critical and denoted by $\sigma_{c}(i)$. In fact, for each $\sigma_{c}(i)$, the total density is constant for the two successive times of iteration $t_{i}$ and $t_{i+1}$.

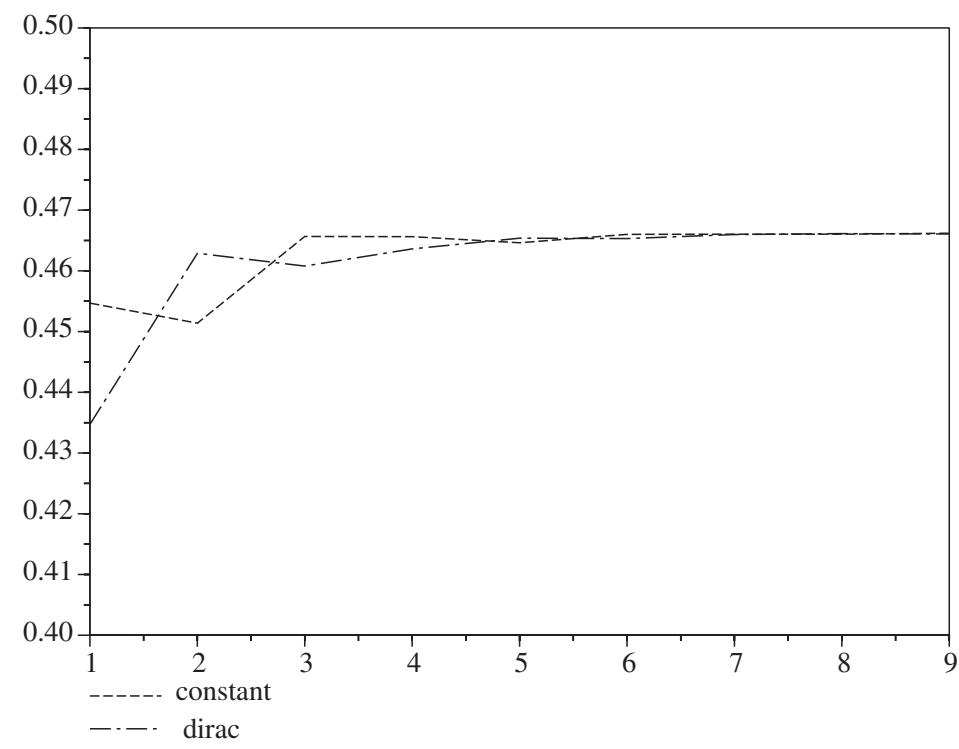

Figure 5. The interpolation curve of $\sigma_{c}(i)$. Each point is given by the intersection of the total density curves for two successive iteration times, i.e. 1 corresponds to the intersection point of 50 and 100 iteration curves. We increase of 50 iterations at every ordinate point, finishing by 500 iterations. 
We observe in Fig. 5 that these values of critical absorption cross sections numerically converge to a finite value $\sigma_{n}$, which is the searched value. Moreover, additional numerical simulations show that this value $\sigma_{n}$ does not depend on $\varepsilon$ (for sufficiently small values of $\varepsilon$ ).

In Fig. 6, we show the limit profile of the curve $n(\cdot, t)$ for the computed critical absorption $\sigma=\sigma_{n}=0.466$ and $t=500 \Delta t$. Again the initial condition is given by a constant function or a Dirac mass. We underline the fact that for both initial data, we end up with the same (up to a normalization) profile which does not depend on $\varepsilon$, for $\varepsilon$ small enough.

In Fig. 7, we show the influence of multiplying reflection boundary conditions with respect to different values of the absorption cross section. When time evolves, we clearly see the significant growth of the number of particles $N(t)$ when $\sigma<\sigma_{n}$, and its fast shoot to zero for $\sigma>\sigma_{n}$. For $\sigma=\sigma_{n}$, this number remains bounded without "exploding" both in the case of the initial condition given by a constant function or a Dirac mass. Moreover, it seems to reach an almost constant value. Hence, this numerical approximation $\sigma_{n}$ of the critical cross-section $\sigma_{c}$ done in Fig. 5 seems to be satisfactory.

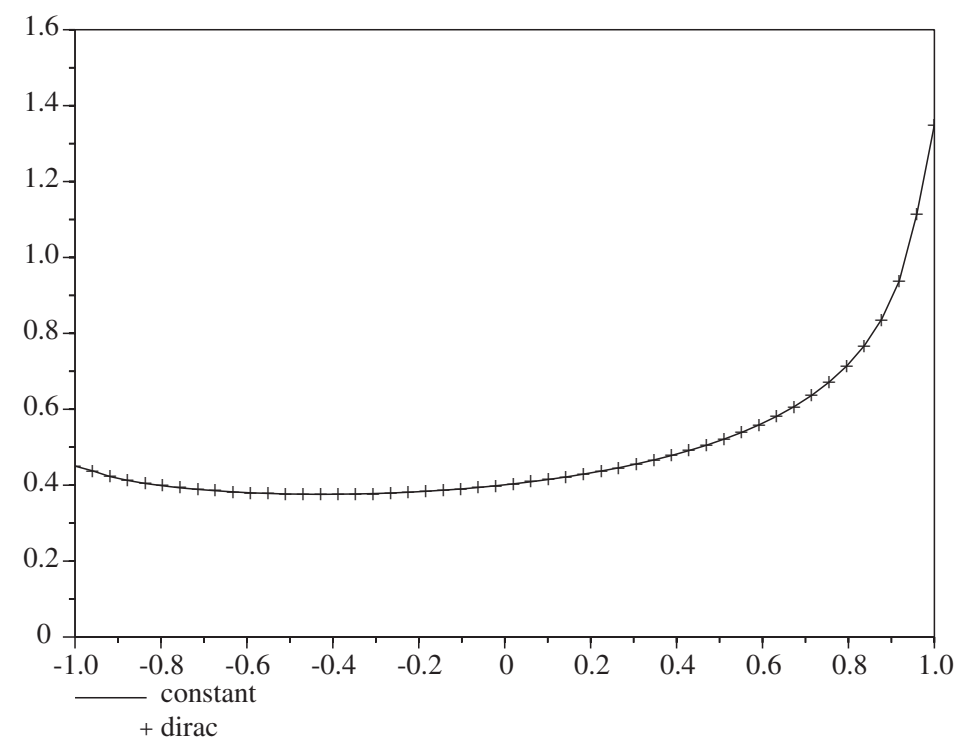

Figure 6. The final profile of $n(\cdot, t)$ with initial data a Dirac mass or a constant function, both in position and velocity; $\sigma=0.466, \varepsilon=0.001, \alpha_{R}=2 \alpha_{L}=4$, $t=500 \Delta t$. 


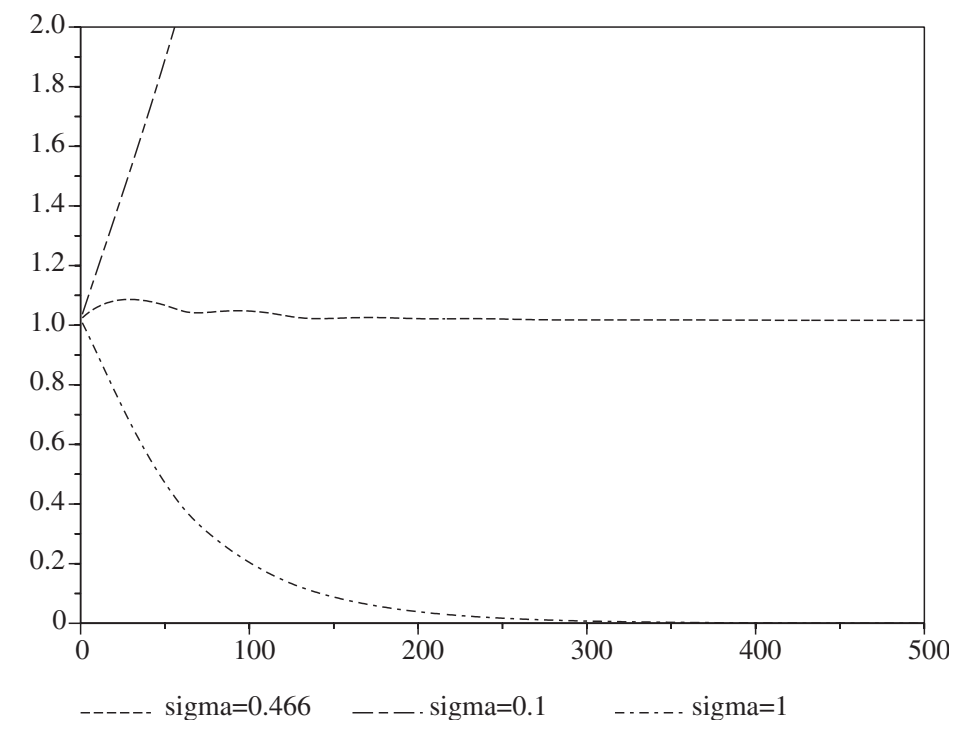

Figure 7. The total density $N(t)$ for different values of the absorption $\left(\sigma>\sigma_{n}\right.$, $\sigma<\sigma_{n}$, and $\left.\sigma=\sigma_{n}=0.466\right)$ with initial data a constant function, $\varepsilon=0.001$ and $t=500 \Delta t$

Finally, in Fig. 8, we show the behavior of the density function $n(\cdot, t)$ for three different iteration times. The initial data which we consider is a Dirac mass both in space and velocity. The absorption cross section $\sigma$ is the numerical critical coefficient $\sigma_{n}=0.466$. It is clearly seen that the Dirac mass is convected and diffused respectively by means of the transport equation and of the Boltzmann-Lorentz operator for grazing collisions (with $\varepsilon=0.001$ ). Finally, the density is also multiplied and reflected at the boundaries, and we can see the beginning of boundary layers.

\subsection{Defocusing of a Beam}

Following the test performed in Cordier et al. (2000) for a photonic type model, we now assume that between the two plates there is vacuum. Moreover, a focussed beam enters in the slab on the left side (i.e., at $x=-L)$ and scatters. The absorption cross section is fixed to $\sigma=0$, the grazing coefficient to $\varepsilon=0.001$ and the discretization points in $x$ and $\theta$ respectively to $N_{x}=50$ and $N_{\theta}=50$. We now consider the influence of the relaxation time $\tau$. The boundary condition corresponding to 
715

716

717

718

719

720

721

722

723

724

725

726

727

728

729

730

731

732

733

734

735

736

737

738

739

740

741

742

743

744

745

746

747

748

749

750

751

752

753

754

755

756

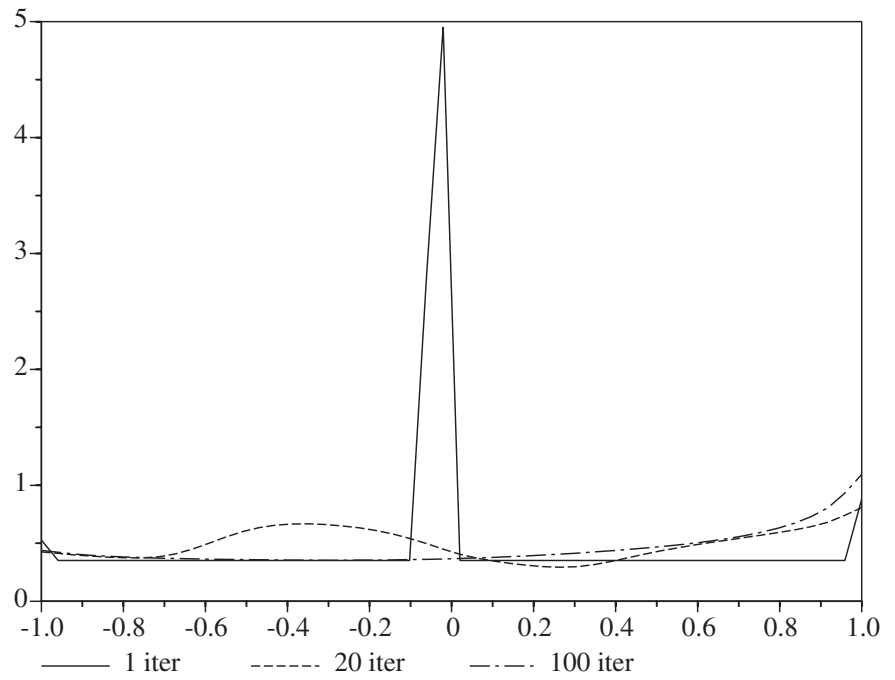

Figure 8. The evolution of $n(\cdot, t)$ with initial data a Dirac mass (normalized) in position and velocity.

the injection of a mono-kinetic beam in the direction perpendicular to the planes of the slab is given by:

$$
f(-L, \cos \theta>0, t)=\delta,
$$

where $\delta$ denotes the delta measure with respect to the angle variable $\theta$; on the right boundary we have:

$$
f(L, \cos \theta<0, t)=0 \text {, }
$$

which means that the particles leaving the slab on the right side never return.

In Fig. 9, we draw the focalization coefficient, defined as the ratio between the particles leaving the slab on the right side with a velocity perpendicular to the plane, and the injected particles:

$$
\mathcal{F}(\tau, t)=f(L, \theta=0, t) .
$$

The computations are done for $t$ sufficiently large $(t=150 \Delta t)$ so that the density has reached a stationary state.

Finally, in Fig. 10 we show for three values of the relaxation time, $\tau=2^{-5}, 1,2^{5}$, and for four iteration times $N_{\text {iter }}=25,50,100,200$ the particle density $n(\cdot, t)$ evolution (with $t=\Delta t N_{\text {iter }}$ ).

Let us note that the numerical results we have obtained for this model are in perfect agreement with those performed in Cordier et al. (2000) 


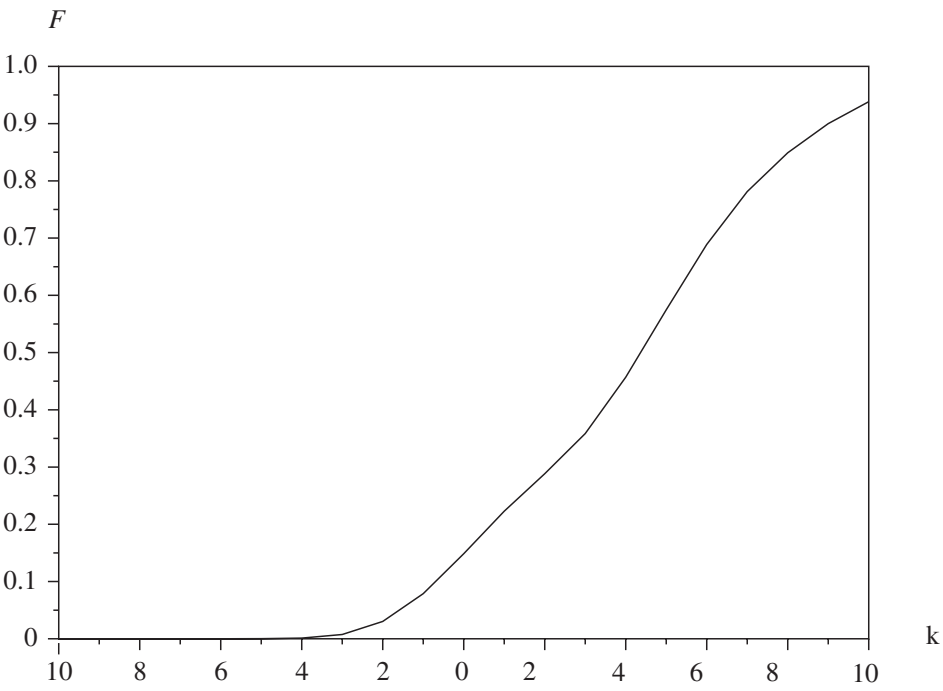

Figure 9. The focalization $\mathcal{F}(\tau, t=150 \Delta t) \cdot \tau=2^{k}, k=-10, \ldots, 10$.

for a $1 \mathrm{D}$ in space and $2 \mathrm{D}$ in velocity (i.e., $R \times S^{2}$ ) analogous problem, where the collision operator involved was the Laplace-Beltrami operator on the unit sphere.

\section{CONCLUDING REMARKS}

We have described an implicit finite element approximation for the grazing collision Boltzmann-Lorentz operator. This approximation allows to pass to the limit $\varepsilon \rightarrow 0$ for a fixed discrete scheme (with respect to the velocity variable). This fact is due essentially to the possibility of carrying out the exact computations of both the mass matrix and the collision matrix with no need of quadrature formulas. Other methods would have been possible: for example, a finite volume method based on a piecewise parabolic operator or a spectral method (see Buet et al. (2001), Pareschi et al. (2002)). Our choice of a FEM method has been essentially motivated by the nature of the limiting problem and also by previous computations done for the Laplace-Beltrami operator in 3D.

The generalization to the three-dimensional case seems to be possible: we can in fact compute the matrices $A$ and $Q$, but the coefficients we find are much more complicated. According to Buet et al. (2001), 
Finite Element Approximation of Grazing Collisions

799

800

801

802

803

804

805

806

807

808

809

810

811

812

813

814

815

816

817

818

819

820

821

822

823

824

825

826

827

828

829

830

831

832

833

834

835

836

837

838

839

840
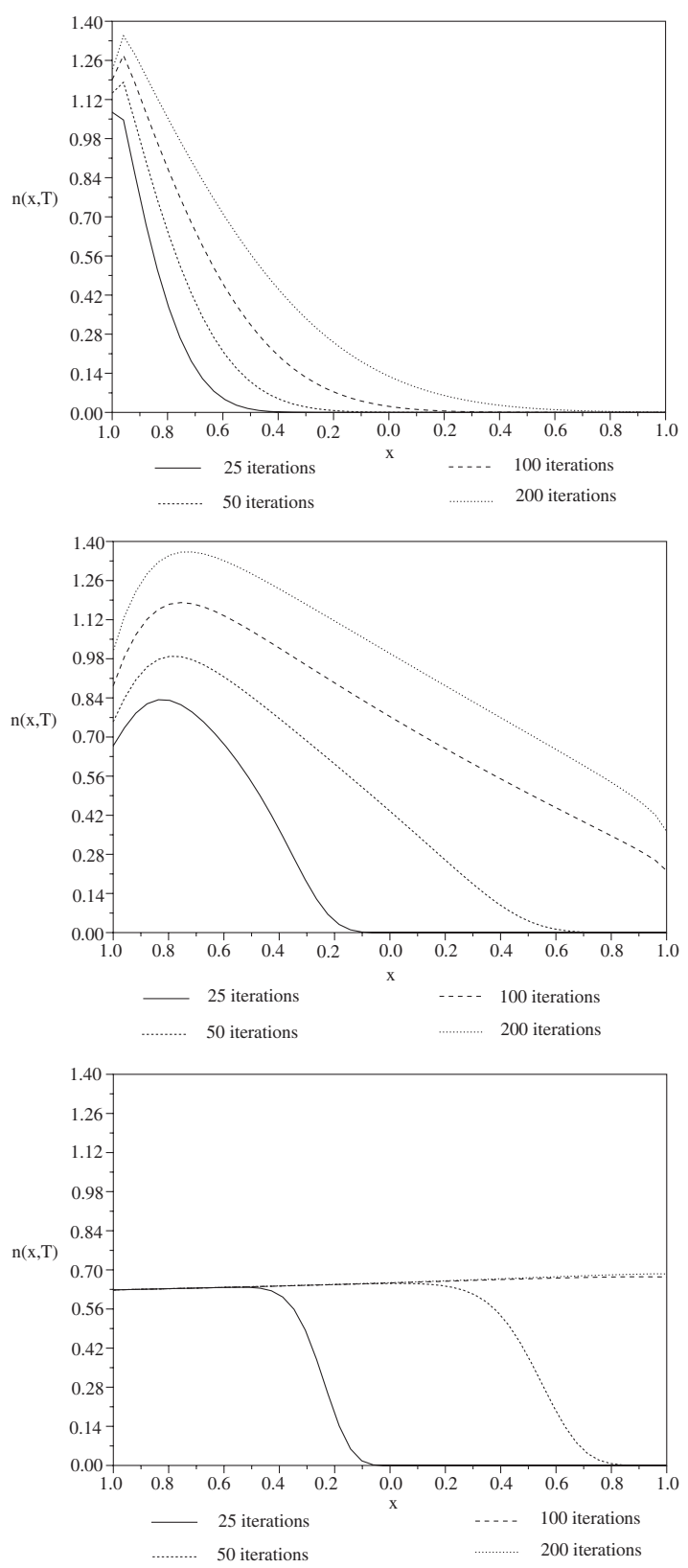

Figure 10. The density $n(\cdot, T), \tau=2^{-5}$ (left), 1 (center), $2^{5}$ (right), $T=N_{\text {iter }} \Delta t$ with $N_{\text {iter }}=25,50,100,200$. 
841

\section{A1. APPENDIX}

Here we perform the computation of the coefficients $Q_{i j}$ of the grazing collision matrix $Q$. We first express the hat functions $\varphi_{i}$ as follows: $\varphi_{i}(\theta)=\varphi_{h}\left(\theta-\theta_{i}\right)$, where:

$$
\varphi_{h}(z)= \begin{cases}h^{-1}(h-|z|), & \text { if }|z|<h, \\ 0 & \text { else. }\end{cases}
$$

Replacing in Eq. (3) $\bar{\theta}=\theta-\theta_{i}$ and $\bar{\theta}^{\prime}=\theta^{\prime}-\theta_{j}$ yields:

$$
Q_{i j}=\int_{S^{1}} \int_{S^{1}} B^{\varepsilon}\left(\bar{\theta}-\bar{\theta}^{\prime}+\Delta \theta\right)\left[\varphi_{h}(\bar{\theta}) \varphi_{h}\left(\bar{\theta}^{\prime}\right)-\varphi_{h}(\bar{\theta}) \varphi_{h}(\bar{\theta}+\Delta \theta)\right] d \bar{\theta} d \bar{\theta}^{\prime},
$$

where we have set $\Delta \theta=\theta_{i}-\theta_{j}=(i-j) h$. Introducing the new hat function

$$
\varphi(z)= \begin{cases}(1-|z|), & \text { if }|z|<1, \\ 0 & \text { else }\end{cases}
$$

we have $\varphi_{h}(z)=\varphi(z / h)$. Now using the new variables $\theta=\bar{\theta} / h$ and $\theta^{\prime}=\bar{\theta}^{\prime} / h$, we can write:

$$
\begin{aligned}
Q_{i j}= & h^{2} \int_{-1}^{+1} \int_{-1}^{+1} B^{\varepsilon}\left(h \theta-h \theta^{\prime}+\Delta \theta\right)\left[\varphi(\theta) \varphi\left(\theta^{\prime}\right)\right. \\
& -\varphi(\theta) \varphi(\theta+(i-j))] d \theta d \theta^{\prime} \\
= & h^{2} \int_{-1}^{+1} \int_{-1}^{+1} B^{\varepsilon}\left(h\left(\theta-\theta^{\prime}+(i-j)\right)\right)\left[\varphi(\theta) \varphi\left(\theta^{\prime}\right)\right. \\
& -\varphi(\theta) \varphi(\theta+(i-j))] d \theta d \theta^{\prime} .
\end{aligned}
$$

Let us set $B_{h}(z)=B(h z)$, i.e., (with $B_{0}=1 / \varepsilon^{3}$ ):

$$
B_{h}(z)= \begin{cases}B_{0} & \text { if }|z|<\alpha=\varepsilon \pi / h \\ 0 & \text { else }\end{cases}
$$


then the collision coefficient $Q_{i j}$ reads:

$$
\begin{aligned}
Q_{i j}= & h^{2} \int_{-1}^{+1} \int_{-1}^{+1} B_{h}\left(\theta-\theta^{\prime}+(i-j)\right)\left[\varphi(\theta) \varphi\left(\theta^{\prime}\right)\right. \\
& -\varphi(\theta) \varphi(\theta+(i-j))] d \theta d \theta^{\prime} \\
= & h^{2} B_{0} \iint_{\left|\theta-\theta^{\prime}+(i-j)\right|<\alpha}\left[\varphi(\theta) \varphi\left(\theta^{\prime}\right)-\varphi(\theta) \varphi(\theta+(i-j))\right] d \theta d \theta^{\prime}
\end{aligned}
$$

with $\theta, \theta^{\prime} \in[-1,+1]$. We split $Q_{i j}$ into a gain part $G_{i j}$ and a loss part $L_{i j}$, i.e., we set $Q_{i j}=G_{i j}-L_{i j}$, where:

$$
\begin{aligned}
G_{i j} & =h^{2} B_{0} \iint_{\left|\theta-\theta^{\prime}+(i-j)\right|<\alpha} \varphi(\theta) \varphi\left(\theta^{\prime}\right) d \theta d \theta^{\prime}, \\
L_{i j} & =h^{2} B_{0} \iint_{\left|\theta-\theta^{\prime}+(i-j)\right|<\alpha} \varphi(\theta) \varphi(\theta+(i-j)) d \theta d \theta^{\prime} .
\end{aligned}
$$

We now have to compute separately these gain and loss terms. For this, we will assume that $\alpha \leq 1$, so that $\varepsilon \leq h / \pi=2 / N_{\theta}$ : it is thus possible to pass to the limit first for $\varepsilon \rightarrow 0$ and then for $h \rightarrow 0$.

Let us first compute the gain coefficients $G_{i j}$. As the $\varphi$ function is null outside a domain of length 2 , it is clear that for $|i-j| \geq 3, G_{i j}=0$ (see also Fig. 11). Thus, we have to compute $G_{i j}$ for $i=j,|i-j|=1$, and $|i-j|=2$. Considering also the symmetry of the collision operator, we only have to compute the integrals for $i-j=-2, i-j=-1$, and $i=j$.

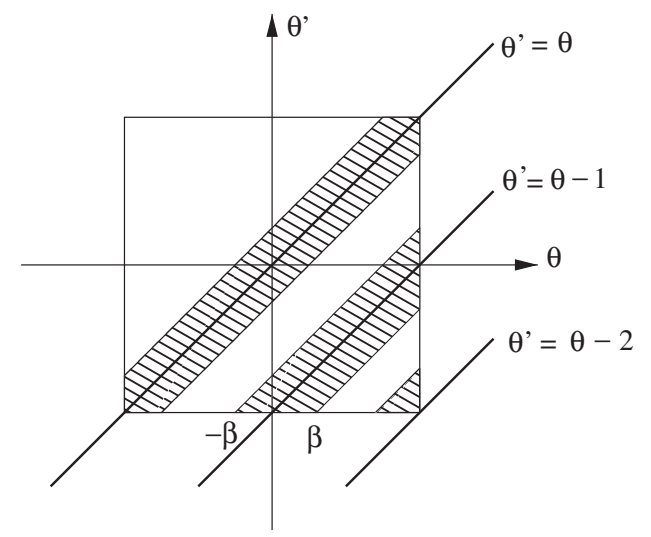

Figure 11. Set of definition of $B_{h}\left(\theta-\theta^{\prime}+i-j\right)$. 
960

961

962

963

964

965

966

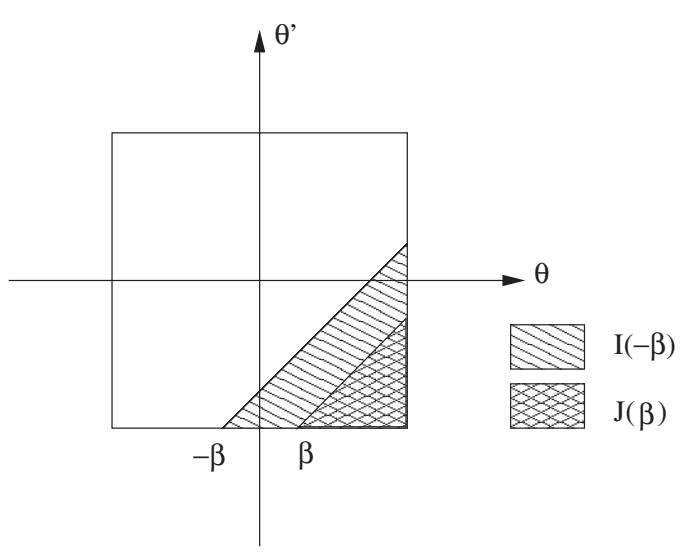

Figure 12. The integrals $I(-\beta)$ and $J(\beta)$. $\beta>0$ :

Looking at Fig. 12, we define the following integrals for a given $\quad$ F12

$$
\begin{aligned}
I(-\beta)= & h^{2} B_{0} \int_{-\beta}^{0}(1+\theta)\left(\int_{-1}^{\theta+\beta-1}\left(1+\theta^{\prime}\right) d \theta^{\prime}\right) d \theta \\
& +h^{2} B_{0} \int_{0}^{1-\beta}(1-\theta)\left(\int_{-1}^{\theta+\beta-1}\left(1+\theta^{\prime}\right) d \theta^{\prime}\right) d \theta \\
& +h^{2} B_{0} \int_{1-\beta}^{1}(1-\theta)\left(\int_{-1}^{0}\left(1+\theta^{\prime}\right) d \theta^{\prime}\right) d \theta \\
& +h^{2} B_{0} \int_{1-\beta}^{1}(1-\theta)\left(\int_{0}^{\theta+\beta-1}\left(1-\theta^{\prime}\right) d \theta^{\prime}\right) d \theta \\
= & h^{2} B_{0}\left(\frac{1}{12} \beta^{3}(\beta-4)+\frac{1}{24}(1-\beta)(1+\beta)\left(\beta^{2}+4 \beta+1\right)+\frac{1}{4} \beta^{2}\right) \\
= & h^{2} B_{0}\left(-\frac{1}{8} \beta^{4}+\frac{1}{6} \beta^{3}+\frac{1}{4} \beta^{2}+\frac{1}{6} \beta+\frac{1}{24}\right)
\end{aligned}
$$

and

$$
\begin{aligned}
J(\beta) & =h^{2} B_{0} \int_{\beta}^{1}(1-\theta)\left(\int_{-1}^{\theta-\beta-1}\left(1+\theta^{\prime}\right) d \theta^{\prime}\right) d \theta \\
& =h^{2} B_{0} \frac{1}{24}(\beta-1)^{4}
\end{aligned}
$$


1001

1002

1003

1004

1005

1006

1007 1008

In particular, $I(-\beta)$ represents the integral with support on the bigger triangle and $J(\beta)$ represents the integral with support on the small triangle (Fig. 12)

We get:

$$
\begin{aligned}
G_{i j} & =J(1-\alpha)=\frac{1}{24} h^{2} B_{0} \alpha^{4}, \quad \text { for } i-j=-2 \\
G_{i j} & =I(-\alpha)-J(\alpha)=h^{2} B_{0}\left(-\frac{1}{6} \alpha^{4}+\frac{1}{3} \alpha^{3}+\frac{1}{3} \alpha\right), \text { for }|i-j|=1 \\
G_{i j} & =h^{2} B_{0}\left(\int_{-1}^{+1}(1-|\theta|) d \theta\right)\left(\int_{-1}^{+1}\left(1-\left|\theta^{\prime}\right|\right) d \theta^{\prime}\right)-2 I(-1+\alpha) \\
& =h^{2} B_{0}\left(\frac{1}{4} \alpha^{4}-\frac{2}{3} \alpha^{3}+\frac{4}{3} \alpha\right), \text { for } i=j
\end{aligned}
$$

We now compute the loss coefficients $L_{i j}$. As before, we just have to compute $L_{i j}$ for $|i-j|=2,1$, and 0 . We first remark that for $|i-j| \geq 2$, the product $\varphi(\theta) \varphi(\theta+i-j)$ is null. In fact $\varphi(\theta)$ differs from zero on the set $[-1,+1]$ while $\varphi(\theta+i-j)$ differs from zero on the set [1,3]. Hence, $L_{i j}=0$ for $|i-j| \geq 2$. Moreover, for $|i-j|=\mathbf{1}$ the product $\varphi(\theta) \varphi(\theta+i-j)$ is not null only for $\theta \in[0,1]$, and the dependence of $L_{i j}$ on the variable $\theta^{\prime}$ appears only through the kernel $B_{h}$ which is not null on a set of amplitude $2 \alpha$. Thus, we have:

$$
\begin{aligned}
L_{i j} & =h^{2} B_{0} 2 \alpha \int_{0}^{1} \theta(1-\theta) d \theta \\
& =h^{2} B_{0} 2 \alpha \frac{1}{6}=\frac{1}{3} h^{2} B_{0} \alpha, \quad \text { for }|i-j|=\mathbf{1}
\end{aligned}
$$

Analogously, for the principal diagonal, we have:

$$
\begin{aligned}
L_{i i} & =h^{2} B_{0} 2 \alpha \int_{-1}^{+1}(1-|\theta|)^{2} d \theta \\
& =h^{2} B_{0} 2 \alpha \frac{2}{3}=\frac{4}{3} h^{2} B_{0} \alpha .
\end{aligned}
$$

Thus, collecting Eqs. (19)-(23) and recalling that $B_{0}=1 / \varepsilon^{3}$ and that $\alpha=\varepsilon \pi / h$, the computation of the coefficients $Q_{i j}$ is completed. 
We finally remark that the computation of the coefficients $A_{i j}$ of the mass matrix $A$ may be done analogously.

\section{ACKNOWLEDGMENT}

We would like to thank the referees for their very helpful comments. The authors also thank the European TMR Network "Asymptotics methods in kinetic theory" $N^{o}$ ERB FMR XCT 970157 for its financial support. This research was supported through a European Community Marie Curie Fellowship.

\section{REFERENCES}

Arlotti, L., Bellomo, N., Lachowicz, M. (2000). Kinetic equations modeling populations dynamics. Transp. Theor. Stat. Phys. 29 (1-2):125-139.

Belleni-Morante, A., Moro, A. (1996). Time dependent photon transport in an interstellar cloud with stochastic clumps. Transp. Theor. Stat. Phys. 25(1):85-101.

Belleni-Morante, A., Totaro, S. (1996). The successive reflection method in three-dimensional particle transport. J. Math. Phys. 37(6): 2815-2823.

Buet, C., Cordier, S., Lucquin-Desreux, B. (2001). The grazing collision limit for the Boltzmann-Lorentz model. Asymp. Analysis 25(2): 93-107.

Cercignani, C. (1988). The Boltzmann Equation and its Applications. New York: Springer.

Cordier, S., Lucquin-Desreux, B., Mancini, S. (2002). Focalization: a numerical test for smoothing effects of collision operators. Preprint N.02-05, http://www.univ-orleans.fr/SCIENCES/MAPMO/publications.

Cordier, S., Lucquin-Desreux, B., Sabry, A. (2000). Numerical method for Vlasov-Lorentz models. ESAIM Proceed. CEMRACS 1999. http://www.emath.fr/Maths/Proc/Vol.10.

Degond, P., Lucquin-Desreux, B. (1992). The Fokker-Planck asymptotics of the Boltzmann collision operator in the Coulomb case. Math. Mod. Meth. Appl. Sci. 2(2):167-182.

Degond, P., Lucquin-Desreux, B. (1996). The asymptotics of collision operators for two species of particles of disparate masses. Math. Mod. Meth. Appl. Sci. 6(3), 405-436. 
1073

1074

1075

1076

1077

1078

1079

1080

1081

1082

1083

1084

1085

1086

1087

1088

1089

1090

1091

1092

Desvillettes, L. (1992). On asymptotics of the Boltzmann equation when collisions become grazing. Transp. Theor. Stat. Phys. 21(3): 259-276.

Goudon, T. (1997a). Sur l'équation de Boltzmann homogéne et sa relation avec l'équation de Landau-Fokker-Planck: influence des collisions rasantes. C. R. Acad. Sci. Paris, Série 1 265-270.

Goudon, T. (1997b). On Boltzmann equations and Fokker-Planck asymptotics: influence of grazing collisions. J. Stat. Phys. 89(3-4):751-776.

Guérin, H., Méléard, S. (2002). Convergence from Boltzmann to Landau process with soft potential and particle approximations. Preprint $\mathrm{N}$ 698, http://www.proba.jussieu.fr/mathdoc/preprints/index.html.

Lucquin-Desreux, B. (2000). Diffusion of electrons by multicharged ions. Math. Models Methods Appl. Sci. 10(3):409-440.

Mancini, S., Totaro, S. (1998). Transport problems with nonhomogeneous boundary conditions. Transp. Theor. Stat. Phys. 27(3-4): 371-382.

Markowich, P. A., Ringhofer, C. A., Schmeiser, C. (1994). Semiconductor Equations. Springer Verlag.

Pareschi, P., Toscani, G., Villani, C. (2002). Spectral methods for the noncut-off Boltzmann equation and numerical grazing collision limit. Numerische. Mathematik.

Received September 13, 2001

Revised January 31, 2003

Accepted February 4, 2003 
